\title{
Trekker: A Single App for all your Treks
}

Marita S.

B.E. Student

St. Francis Institute of Technology

Mumbai, India
Anu A.

B.E. Student

St. Francis Institute

of Technology

Mumbai, India

\author{
Makarand T. \\ B.E. Student \\ St. Francis Institute \\ of Technology \\ Mumbai, India
}

\author{
Supriya Y. \\ B.E. Student \\ St. Francis Institute \\ of Technology \\ Mumbai, India
}

\author{
Vipula R. \\ Assistant Professor \\ St. Francis Institute \\ of Technology \\ Mumbai, India
}

\begin{abstract}
Trekking is an activity in which people take hiking trips through rural, often rugged territory. As travelers move through rural areas, trekking gives them an up-close view of the scenery. There are innumerable trekking places in the state of Maharashtra. Some spots are well known whereas some are still isolated. The information required by a trekker includes locating nearby trekking spots, the difficulty level involved in trekking, reviews of the people who have already been there, directions and the climatic condition of the place. In this paper, an android application that helps one to easily discover unusual trekking locations that are otherwise not much talked about, mentioned or is difficult to discover is introduced. This is a completely user friendly application, providing the best destination match and is more convenient than referring to any travel book or map.
\end{abstract}

\section{General Terms}

Android Application

\section{Keywords}

Global Positioning System (GPS), Android App, Trekking, User Friendly, Ionic, Cordova.

\section{INTRODUCTION}

Trekking is an adventurous journey to discover the diversity of nature and culture by foot on hills or high mountain areas which is yet not accessible by modern transportation. Due to the modern hectic lifestyle, need for relaxing and distressing is felt. Travelling has always been the best option to relax. TREKKER is an application which is specially designed for this purpose allowing trekkers to detect their current location using Global Positioning System (GPS) available in the android phones. Accordingly, it provides the nearest trekking locations based on their current position and sorts the areas according to their climatic conditions, difficulty level etc. It allows one to share their experience with other users as well. It gives useful information about the desired trekking spot such as images and overview of the spots. One can also upload photos or post reviews for that place so that they can in turn help out other trekkers to review the place before they go out on their trekking expedition. The focus of this study is to create an application which helps one to easily discover rare and unique trekking locations, provide crucial information to appease the habit of sharing socially, save searching time by entering location manually so as to have an easy to use quick app.

\section{LITERATURE REVIEW}

Sawsan Alshattnawi's and et.al paper gives a great overview about android framework which has helped in understanding the fundamentals of android platform. It also provides proper and systematic working of the tourist guide program which aids to conceive the back-end working. It has helped in understanding the stages at which user can store his feedback and images into the database [1].

Prof. Seema Vanjire's and et.al paper helps to understanding the importance of an android application that gives the users real time and exact information. It also adds a lot of information about the basic working of the GPS. For tracking the user's current location in the application the same has been used. Google Application Program Interface (API) is pivotal in plotting the trekking spots on the map [2].

The use of climatic knowledge in the application is an important idea given in by Bagrecha Komal and et.al. Whenever user plans to go for a trek, the user should be aware about the climate, as it can affect the trekking experience. For this purpose, the climate details for every location that in the application covers have been stored into the database [3].

\section{PROPOSED WORK}

An application that allows users to access all the useful information at their fingertips has been proposed. It is developed on an android platform which is user friendly and reliable.

\subsection{Scope of the Project}

TREKKER will provide the users with an easy experience of trekking where they can always rely on the app for choosing the perfect trek. This app may or may not cover all the additional places and trekking spots within the boundaries of Maharashtra but with future enhancements and user reviews this shortcoming will also be covered up soon. Sometimes describing a spot perfectly along with all the exact information might prove as a constraint, but the users can be rest assured that there will never be any wrong information provided.

The working of the TREKKER application is shown in Fig. 1. The image indicates an activity and arrows represent the flow. User is the one whose current location is tracked using GPS then nearby trekking places are suggested. A list of available shops for supplies are shown also a navigation is shown from start to end. The user can upload pictures and finally review the place. 


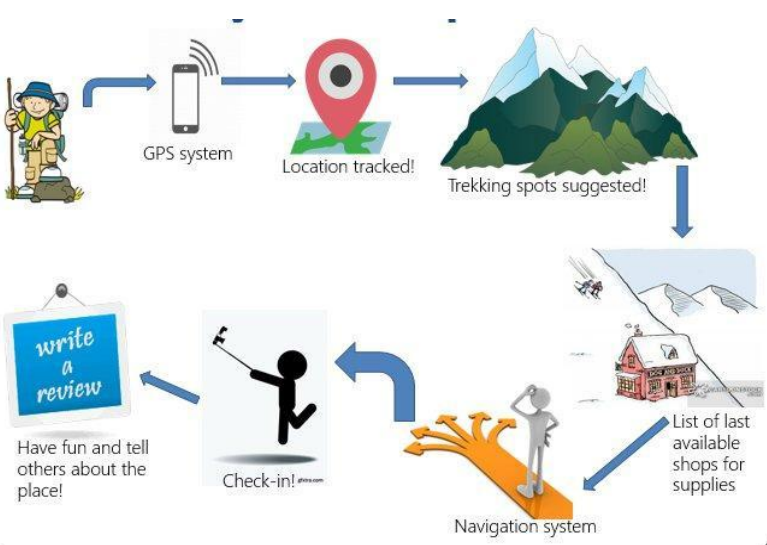

Fig.1 Working of TREKKER Application.

\subsection{Architectural Design}

TREKKER is an android app that takes the user location using GPS and displays the nearby trekking spots. Locations based on favorable climate, trends or reviews can also be viewed. The app also provides useful information about the trekking spots such as its height, distance, difficulty level, location etc. Since, all the transfer of information is between the end user and client, this app has a two tier architecture which is also called as Client/Server Architecture.

In 2-tier Architecture (client/server architecture), the input is given by user and the most relevant and desired output is given by the server. The architecture consists of two tiers, End user and the Server. It has two layers, the first layer is the presentation layer that is the interface, that runs on the client system and the second layer is the Data layer where all the data structure are stored on the server illustrated in Fig. 2.

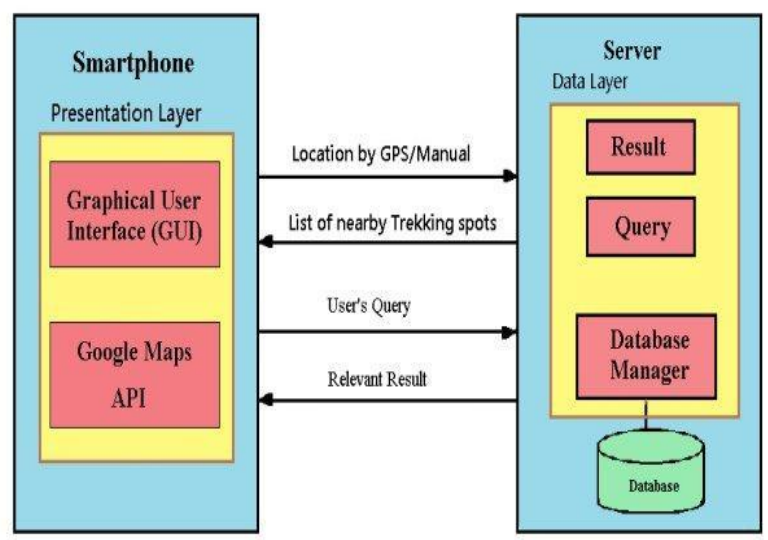

Fig. 2. Two Tier Architecture

The user creates a login Id and password for it. To make sure that the user is legitimate an email is sent to the user through which he/she verifies who he/she claims to be. The user's current location or manual inputs are sent to the server from end user using the GPS technique. The server provides the nearby trekking locations to the end user and also gives useful information about that trekking spot. The user can filter this displayed list of options on the basis of weather, difficulty level, length etc. These filtered inputs are sent to the server. The database is crawled to match the queries of the end user's input and displays the relevant result.

Once the user has chosen the trekking spot, the server then provides the user with the distance to reach the trekking spot. The two tier architecture and user friendly GUI makes it easy for the end users to use the application which saves time as the search is narrowed down thereby reducing efforts. It provides an easy filter which makes it convenient for user to filter out the best location on the basis of weather, duration, distance of the trekking spot at their own leisure. It is a user friendly application since it can be used by anyone from anywhere.

\section{RESULT AND DISSCUSSION}

The tool previously used to make this app was Android Studio [4]. Since android studio was more time consuming, Ionic and Cordova is chosen. The use of Ionic and Cordova reduces the Android Application Package (APK) size there by taking less memory space of the device. [5][6][7]

Software Requirements

- Ionic

- Cordova

- Android SDK tools (JellyBean or Above) Sublime Text 3

- $\quad$ Ripple Emulator

Hardware Requirements

- 2GB RAM or more

- Monitor resolution of 1024 x 768 or higher. Intel Pentium 4 (or above).

- 4 GB (or more) available hard disk space. Android Device

- Cordova

Plug-in

- Geolocation Plug-in: The use of geolocation plug-in in ionic and Cordova helps to display geographical information and embed markers on it [8].

- SQL Lite plug-in: SQL Lite plug-in helps the developer to use Web SQL for accessing the database. Google chrome, opera, safari and android browser support this plug-in [9].

Following figures show the screenshots of the TREKKER app. The register page is shown in Fig. 3 where the user must enter the details to proceed. Once the user has registered he/she is navigated to the login page as shown in Fig. 4. The user then has to enter username and password. If the entered username and the password are valid, the user is directed to the home page as shown in Fig. 5. On clicking "Closest to you" icon it leads to a pop up asking whether or not to allow the GPS as shown in Fig. 6. Once the GPS is allowed it shows the current location and nearby trekking spots. A trek place can be chosen from a list of places as shown in Fig. 7 and further details is shown on choosing the trek place leading to Fig. 8. A map is shown Fig. 9 which gives navigation to the trekking place chosen. Navigation details can be seen as shown in Fig. 10. Reviews about the place can be uploaded as shown in Fig. 11. Users can capture their memory in "My Diary" as shown in Fig. 12. 


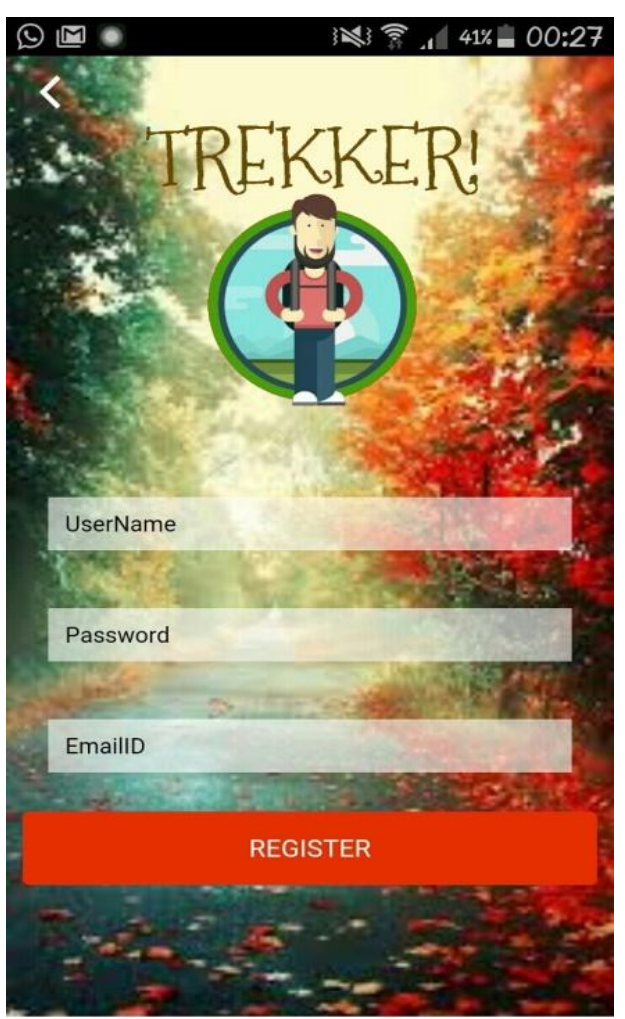

Fig. 3 Register Page

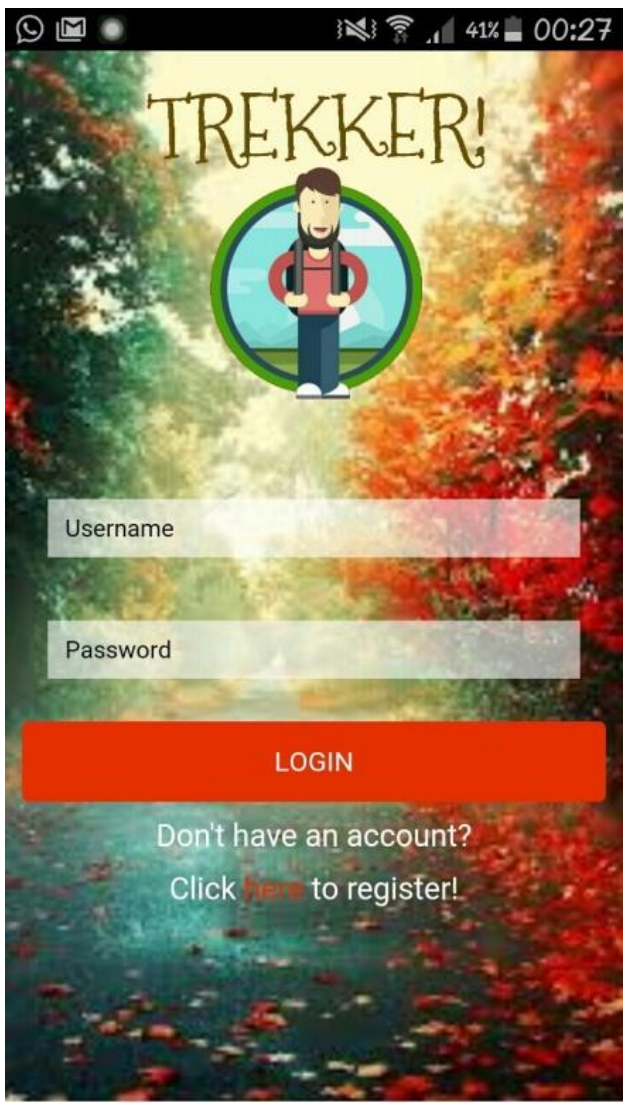

Fig. 4 Login Page

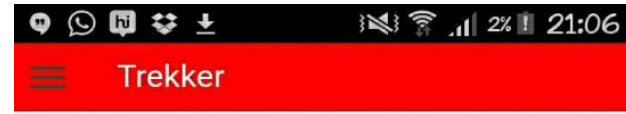
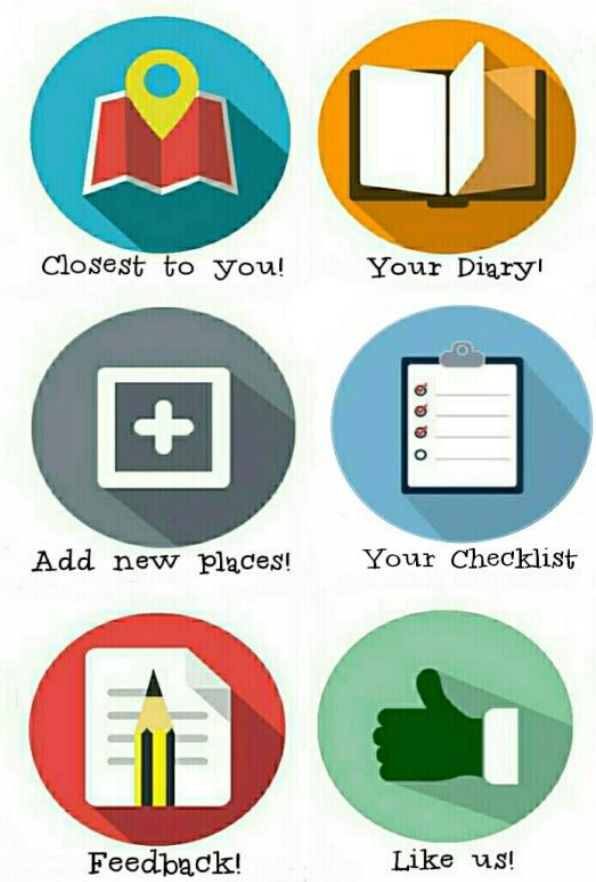

Fig. 5 Home Page

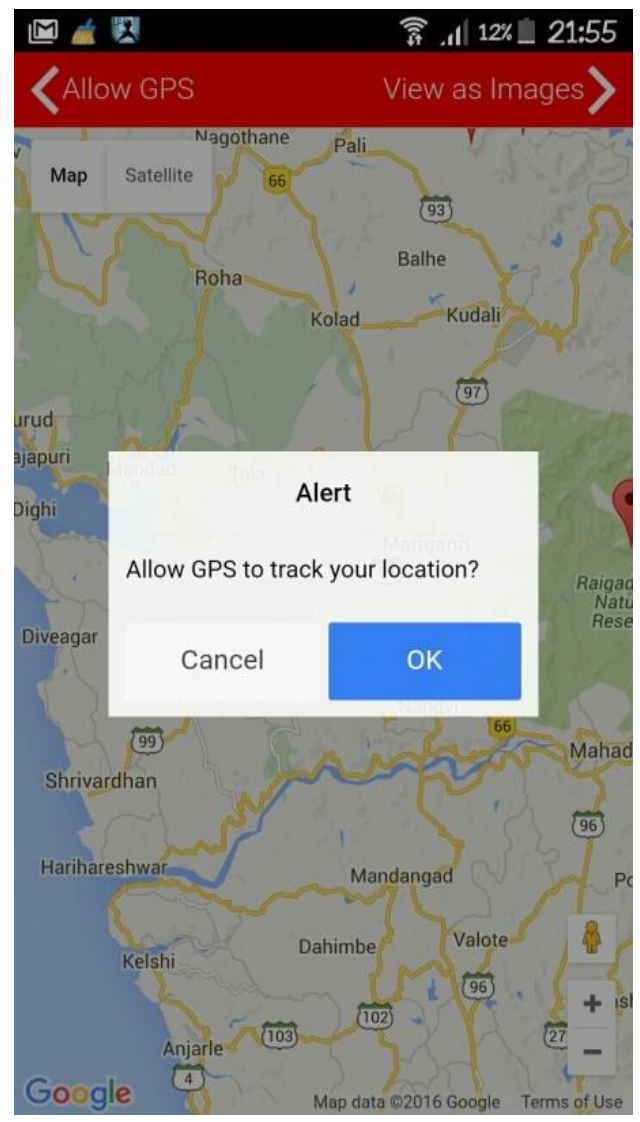

Fig. 6 Allow GPS 


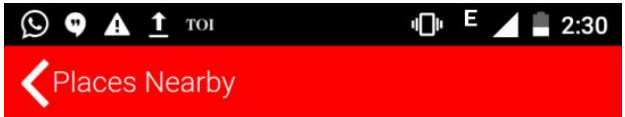

Search Place

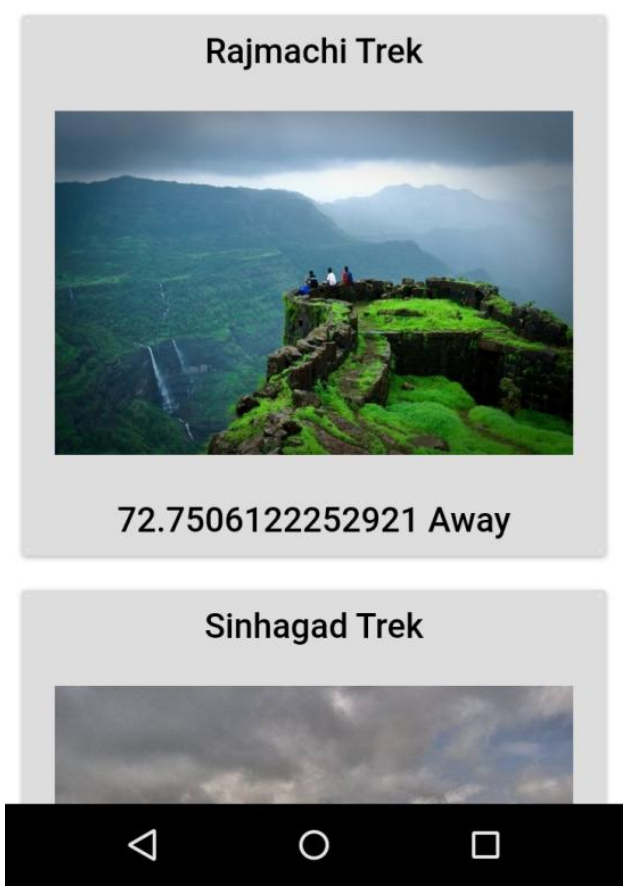

Fig. 7 List of Places

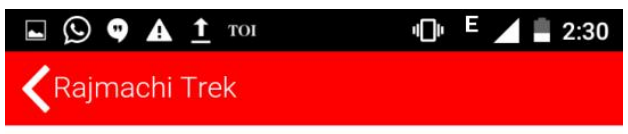

Rajmachi Trek

Iㄴ 72.7506122252921 Away

to All year (monsoon preferred)

A Trek Length: 4-5hrs

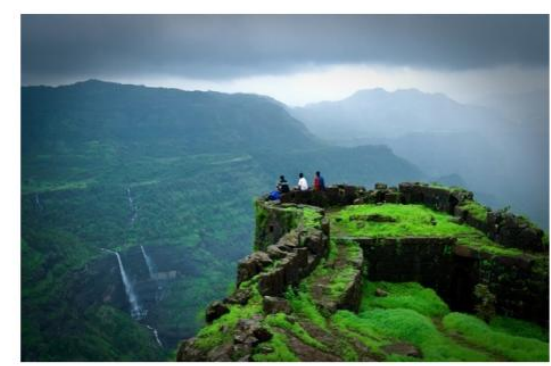

A fort adjoining a historic trade route, Rajmachi $(2,710 \mathrm{ft})$ is one of the most exciting and enduring trekking trails around Pune. An $18 \mathrm{~km}$ trail one

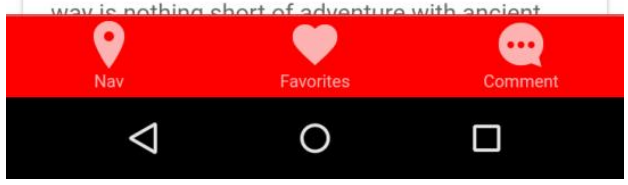

Fig. 8 Place Detail

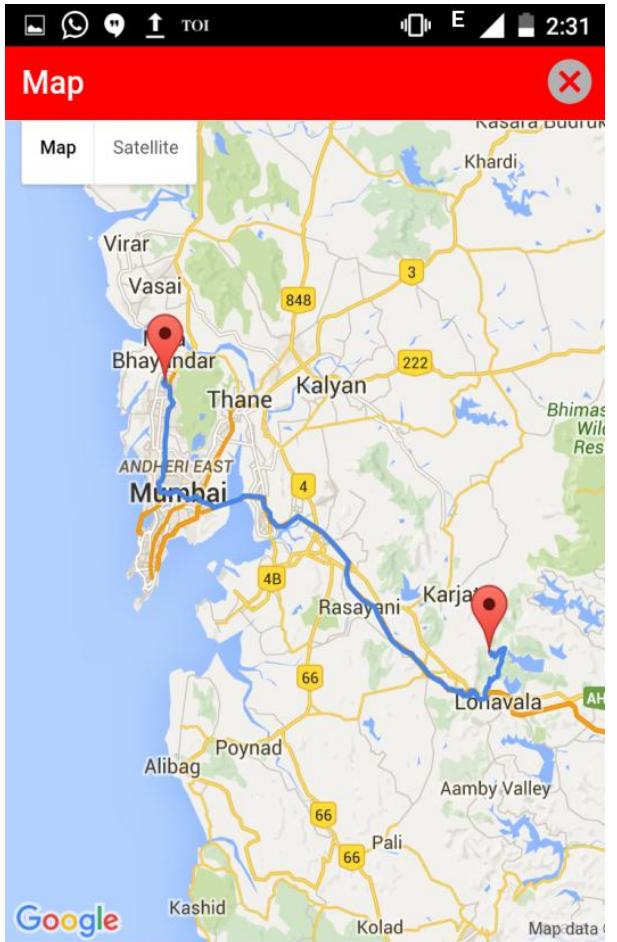

Rajmachi Trek

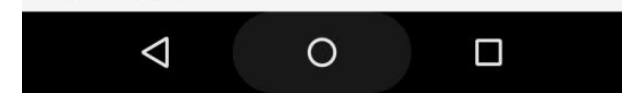

Fig. 9 Navigation

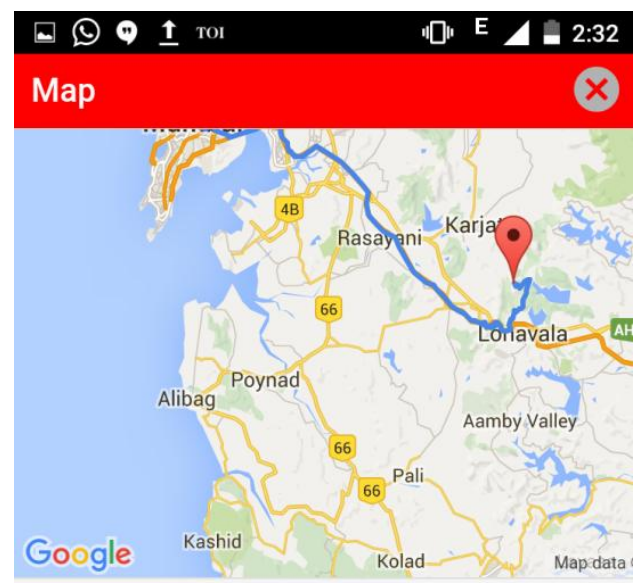

Rajmachi Trek

153.41445289307757 Away from your current location.

A fort adjoining a historic trade route, Rajmachi $(2,710 \mathrm{ft})$ is one of the most exciting and enduring trekking trails around Pune. An $18 \mathrm{~km}$ trail one way is nothing short of adventure with ancient Buddhist caves, waterfalls, lush greenery,

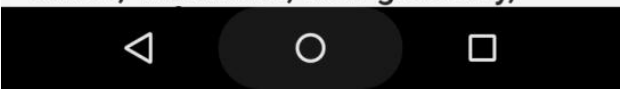

Fig. 10 Navigation Details 


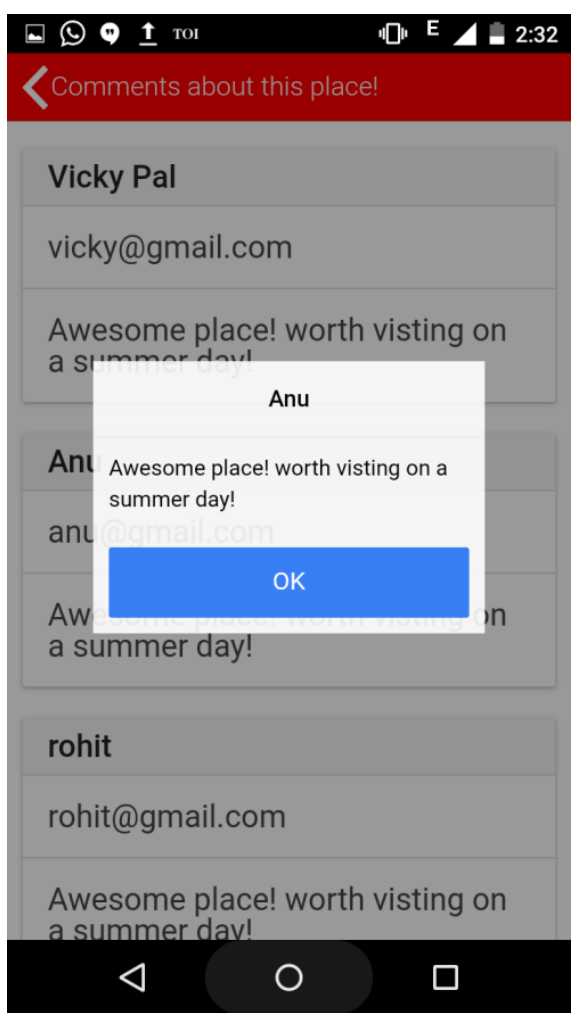

Fig. 11 Comment about a Place

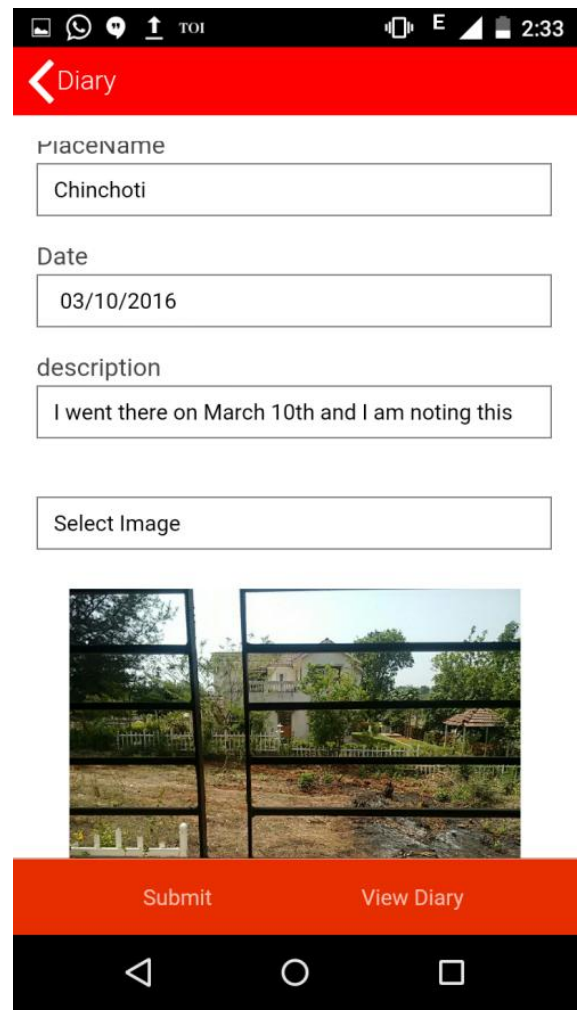

Fig. 12 My Diary

\section{CONCLUSION AND FUTURE SCOPE}

Trekker is thus an application developed on the android platform that basically lets the user choose from a list of various trekking spots in Maharashtra. Once the user searches for a particular spot, the user can view the navigation routes that will make sure the user is always on the right track. The main purpose of this app is to help the trekkers find all the details vital for an enjoyable trekking trip in a single place without surfing through several websites tediously. This app is designed and developed using Ionic and Cordova and will be supported by any Android device. Places outside Maharashtra can also be included to increase the ease of usage for the users thereby providing much wider and better scope for the use of this application. The user interface is designed so as to cater to all the needs of a trekker. It will be made sure that the app is as user friendly as possible so that it can be used by trekkers of all ages.

Currently, TREKKER which is developed using Ionic and Cordova is compatible with Android platform. Further, it can be extended to work on other operating systems such as iOS, Windows and so on.

\section{REFERENCES}

[1] Sawsan A., Amit B., Sneha C., Khivsara," Building Mobile Tourist Guide Applications using Different Development Mobile Platforms" International Journal of Advanced Science and Technology Vol. 54, May, 2013

[2] Prof. Seema V.,Unmesh K., Ganesh S., Pradnyesh P. "Location Based Services on Smart Phone through the Android Application” International Journal of Advanced Research in Computer and Communication Engineering Vol. 3, Issue 1, January 2014

[3] Bagrecha K., Amit B., Sneha, C., Khivsara," Android Application Using GPS Navigation", 1st International Conference on Recent Trends in Engineering \& Technology, Mar-2012 - Special Issue of International Journal of electronics, Communication \& Soft Computing Science \& Engineering, ISSN: 2277-9477

[4] Overview of Android Studio, "http://developer.android.com/tools/studio/index.html" , November 28, 2015

[5] Node.js, "https://nodejs.org/en/about/", January 3, 2016

[6] Overview, "https://cordova.apache.org/" , January 3, 2016

[7] Welcome to ionic, How to get most of Ionic, "http://ionicframework.com/docs/guide/preface.html", January 10, 2016

[8] Cordova SQL Lite, "http://ngcordova.com/docs/plugins/sqlite/",January 10 , 2016

[9] CordovaGPSPlugin,"http://ngcordova.com/docs/plugins/ geolocation/",January 10, 2016 